\title{
Effects of Particulate Matter in a Mouse Model of Oxazolone-Induced Atopic Dermatitis
}

\author{
Yoo Jung Bae*, Kui Young Park*, Hye Sung Han, Young Shin Kim ${ }^{1}$, Ji Yeon Hong ${ }^{2}$, Tae Young Han ${ }^{1}$, \\ Seong Jun Seo \\ Department of Dermatology, Chung-Ang University College of Medicine, ${ }^{1}$ Department of Dermatology, Nowon Eulji Medical Center, \\ Eulji University, ${ }^{2}$ Department of Dermatology, Seoul National University Hospital, Seoul, Korea
}

Background: Recent epidemiological studies have demonstrated that air pollution is associated with the inflammatory response and may aggravate inflammatory skin diseases such as atopic dermatitis (AD). However, it is unclear whether particulate matter (PM) aggravates AD symptoms. Objective: The aim of this study was to investigate whether PM exposure affects the skin barrier dysfunction and aggravates AD symptoms using human keratinocytes (HaCaT) cells and a mouse model of oxazolone-induced AD-like skin. Methods: Standard reference material (SRM) 1649b, which mainly comprises polycyclic aromatic hydrocarbons, was used as the reference PM. HaCaT cells and mouse model of oxazolone-induced AD-like skin were treated with PM. The mRNA or protein expression levels of stratum corneum (SC) and tight junction (TJ) proteins, inflammatory cytokines, as well as clinical and histological changes of the AD-like skin of mouse model were evaluated. The expression of genes and proteins was

Received May 27, 2020, Revised August 7, 2020, Accepted for publication August 13, 2020

*These authors have equally contributed to the article.

Corresponding author: Seong Jun Seo, Department of Dermatology, ChungAng University College of Medicine, 102 Heukseok-ro, Dongjak-gu, Seoul 06973, Korea. Tel: 82-2-6299-1544, Fax: 82-2-6299-1718, E-mail: 626@ caumc.or.kr

ORCID: https://orcid.org/0000-0003-2915-839X

Tae Young Han, Department of Dermatology, Nowon Eulji Medical Center, Eulji University, 68 Hangeulbiseok-ro, Nowon-gu, Seoul 01830, Korea. Tel: 82-2-970-8280, Fax: 82-2-974-1577, E-mail: dermahan@gmail.com ORCID: https://orcid.org/0000-0003-0317-9635

This is an Open Access article distributed under the terms of the Creative Commons Attribution Non-Commercial License (http://creativecommons. org/licenses/by-nc/4.0) which permits unrestricted non-commercial use, distribution, and reproduction in any medium, provided the original work is properly cited.

Copyright (c) The Korean Dermatological Association and The Korean Society for Investigative Dermatology analyzed by real-time polymerase chain reaction and Western blotting. Levels of inflammatory cytokines were measured by enzyme-linked immunosorbent assay. Results: The results revealed that $\mathrm{PM}$ downregulates the expression levels of several SC and TJ-related proteins in the mouse model with AD-like skin. Clinically, epidermal and dermal thickness was significantly increased and dermal inflammation was prominent in PM treated AD-like skin. Conclusion: In conclusion, we found that PM aggravates skin barrier dysfunction, clinically augmenting epidermal and dermal thickening with dermal inflammation in AD-like skin. These results suggest that PM may trigger the exacerbation of AD symptoms via skin barrier dysfunction-related mechanisms. (Ann Dermatol 32(6) $496 \sim 507,2020$ )

\section{-Keywords-}

Air pollution, Atopic dermatitis, Epidermis, Particulate matter

\section{INTRODUCTION}

Particulate air pollutants (particulate matter, PM) are a complex mixture containing various substances such as metals, minerals, organic toxins, and various contaminants (smog, tobacco smoke, pollen, and house dust mite allergens) ${ }^{1-3}$. Ambient PM is known to have harmful effects on human health ${ }^{4-6}$. The World Health Organization estimates that PM contributes to approximately 800,000 premature deaths each year, ranking it the 13th leading cause of mortality worldwide ${ }^{5}$. In particular, ambient PM is highly associated with the morbidity of respiratory and cardiovascular diseases ${ }^{7,8}$. Furthermore, recent epidemiological investigations have demonstrated that PM is also correlated with inflammatory skin diseases such as atopic der- 
matitis (AD), acne, psoriasis, and allergic reactions ${ }^{9}$. The skin is the largest organ in the human body and acts as an essential barrier to protect against harmful external factors such as ultraviolet radiation and ambient $\mathrm{PM}^{10}$. The barrier function of the skin is primarily dependent on two major barrier structures: the stratum corneum (SC), the outermost layer of the epidermis, and the tight junction (TJ) proteins, intercellular junctions that seal adjacent keratinocytes in the stratum granulosum ${ }^{1,11-13}$. However, various environmental pollutants can downregulate the expression of the structural proteins of the SC and TJ proteins $^{3,14-16}$, leading to skin barrier dysfunction and skin inflammation $^{3,16,17}$. Furthermore, PM can penetrate the skin through hair follicles and cause epidermal thickening and dermal inflammation in disrupted skin ${ }^{17,18}$.

$A D$ is a chronic relapsing inflammatory skin disease characterized by complex interactions among genetic predisposition, environmental factors, immune dysregulation, and skin barrier dysfunction ${ }^{19-22}$. Recently, several epidemiologic studies have investigated the effects of PM in patients with $A D^{1,22-26}$. However, the results of these studies are controversial: several studies demonstrated that ambient $\mathrm{PM}$ is related to the exacerbation of $\mathrm{AD}$ symptoms ${ }^{22-24,27}$; in contrast, others report that $A D$ is not related to urbanization and industrialization ${ }^{25,26}$. Moreover, most previous studies primarily focused on the epidemiological perspective; thus, studies on the mechanism by which PM causes or worsens AD symptoms have not been well performed. Therefore, in this study, we aimed to investigate whether PM exposure affects the exacerbation of ADlike symptoms and elucidate the mechanisms by which PM affects AD-like skin.

\section{MATERIALS AND METHODS}

\section{Chemicals and preparation}

The SRMs 1648a and 1649b were purchased from the National Institute of Standards and Technology (Gaithersburg, MD, USA) and dispersed in distilled water. SRM 1648a mainly contains heavy metals, whereas SRM 1649b includes polycyclic aromatic hydrocarbons, polychlorinated biphenyl congeners, and pesticides ${ }^{3}$. Recombinant human interleukin (IL)-4 and IL-13 were purchased from PeproTech (Rocky Hill, NJ, USA). 4-Ethoxymethylene-2-phenyl-2-oxazolin-5-one (OXA) was purchased from Sigma-Aldrich (St Louis, MO, USA). OXA was dissolved in vehicle (acetone: olive oil=4:1 mixture). For western blot analysis, anti-occludin, anti-claudin- 1 , anti-involucrin, and anti- $\beta$-actin antibodies were purchased from Santa Cruz Biotechnology (Santa Cruz, CA, USA). Anti-loricrin and anti-filaggrin were purchased from LifeSpan Biosciences (Seattle, WA, USA).
Anti-E-cadherin and anti-GAPDH antibodies were purchased from Cell Signaling Technology (Danvers, MA, USA). Anti-ZO-1 was purchased from Thermo Fisher Scientific (Waltham, MA, USA). A mouse immunoglobulin E (IgE) ELISA kit was purchased from Thermo Fisher Scientific.

\section{Cell culture}

Human keratinocyte (HaCaT) cells (American Tissue Cell Collection [ATCC] obtained in 2002, USA) were cultured in Dulbecco's modified Eagle's medium (DMEM; Hyclone, Logan, UT, USA) supplemented with $10 \%$ fetal bovine serum (FBS) and $1 \%$ penicillin-streptomycin. Cells were propagated at $37^{\circ} \mathrm{C}$ in a humidified atmosphere of $5 \% \mathrm{CO}_{2}$. When the cultures reached confluence, cells were treated with $0.05 \%$ trypsin/ $0.53 \mathrm{mM}$ EDTA for 5 minutes at $37^{\circ} \mathrm{C}$. $\mathrm{HaCaT}$ cells were used in the experiments between passages 20 to 24 .

\section{HaCaT cells were treated with IL-4, IL-13, and particulate matters}

Since type 2 helper T cell (Th2) cytokines IL-4 and IL-13 have been implicated in the pathogenesis of $\mathrm{AD}, \mathrm{HaCaT}$ cells were treated with $50 \mathrm{ng} / \mathrm{ml}$ concentrations of recombinant human IL-4 and IL-13 to establish an in vitro model of AD. After incubation for 72 hours, the cells were treated with PMs (SRM 1648a and 1649b) at concentrations of $50 \mu \mathrm{g} / \mathrm{cm}^{2}$ and $25 \mu \mathrm{g} / \mathrm{cm}^{2}$, respectively. The plates were then incubated at $37^{\circ} \mathrm{C}$ for 24 hours. After incubation, all of the HaCaT cells, including the control, were evaluated at the same time point. PMs were used at the optimal concentrations mentioned in prior studies ${ }^{28,29}$.

\section{Animals}

Six-week-old female BALB/c mice $(n=5$ in each experimental condition; $\mathrm{n}=20$ in total) were housed under specific pathogen-free conditions and allowed to acclimatize for 1 week before starting the experiment. All animal experiments were performed in accordance with the regulations and upon the approval of the Institutional Animal Care and Use Committee (IACUC) of the Chung-Ang University (IRB Approval No. 2018-00097).

\section{Induction of atopic dermatitis-like skin lesions and exposure to particulate matter}

The mouse model with OXA-induced AD-like skin is a well-known animal model for AD-like symptoms. It is induced by multiple epicutaneous administrations of OXA (Fig. 1A), a potent hapten that induces a chronic Th2 hypersensitivity reaction resembling the features of initial human $A D^{30}$.

The dorsal hair of the mice was shaved 2 days before ini- 
A<smiles>CCOC=C1N=C(c2ccccc2)OC1=O</smiles>

B $B A L B / c$
$n=5$

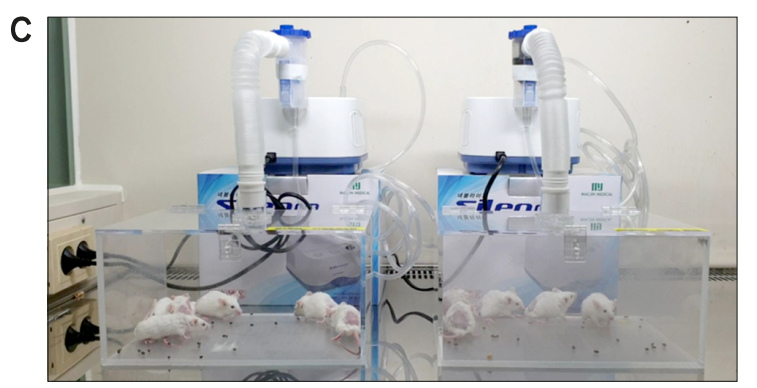

Without PM exposure

PM exposure
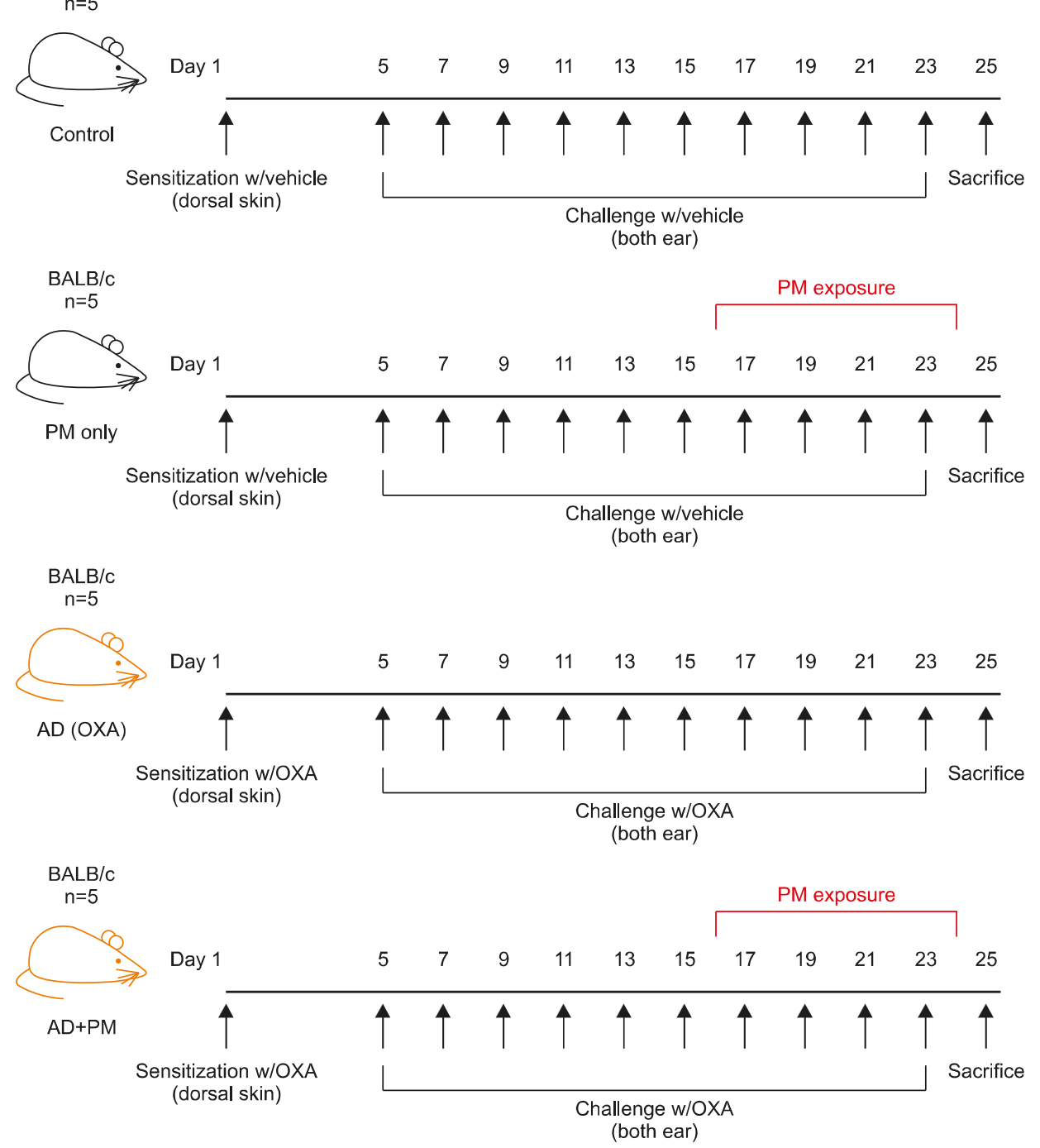

Fig. 1. Experimental protocol of this study. (A) Chemical structure of oxazolone. (B) Experimental protocol for the induction of $A D$-like skin and PM exposure. (C) Closedsystem chamber attached to a nebulizer for PM exposure. AD: atopic dermatitis, PM: particulate matter, OXA: oxazolone.

tiating the process of sensitization. Then, 10 mice each were sensitized with $20 \mu \mathrm{l}$ of $1 \%$ OXA or vehicle applied to the shaved dorsal skin. Four days after sensitization, the ears of 10 mice sensitized with vehicle were repeatedly challenged with vehicle, and the ears of 10 mice sensitized with OXA were repeatedly challenged with $20 \mu$ l of $0.1 \%$ OXA once every other day for 18 days (Fig. 1B). After performing the OXA or vehicle challenge six times, 5 mice from each group were exposed to $100 \mu \mathrm{g} / \mathrm{cm}^{3}$ SRM $1649 \mathrm{~b}$ for $1 \mathrm{~h} /$ day in a closed-system chamber attached to a nebulizer (Macjin Medical, Seoul, Korea), once every other day for 10 days (Fig. 1C). The other 5 mice from each group were left in the same closed-system chamber attached to a nebulizer but without exposing to PM (Fig. 1C).

Ear thickness was measured using a digital caliper (Mitu- 
toyo, Tokyo, Japan) before every challenge and immediately before sacrifice. Ear skin and blood samples were collected after the mice were anesthetized using 2,2,2-tribromoethanol (Avertin; Sigma-Aldrich). OXA treatment was performed and SRM 1649b exposure conditions were in accordance with previously described protocols with modifications ${ }^{21,30-33}$.

\section{RNA extraction and quantitative real-time PCR analysis}

Total RNA was extracted from the cells and ear tissues using TRIzol ${ }^{\circledR}$ reagent (Welgene, Seoul, Korea) according to the manufacturer's protocol. cDNA templates were synthesized from mRNA by reverse transcription using a RevertAid First Strand cDNA Synthesis Kit (Thermo Fisher Scientific) and incubated for 1 hours at $42^{\circ} \mathrm{C}$. The resultant CDNA was then amplified by quantitative real-time PCR (qPCR) with primers specific for IL- $1 \alpha$, IL-1 $\beta$, IL-4, IL-6,
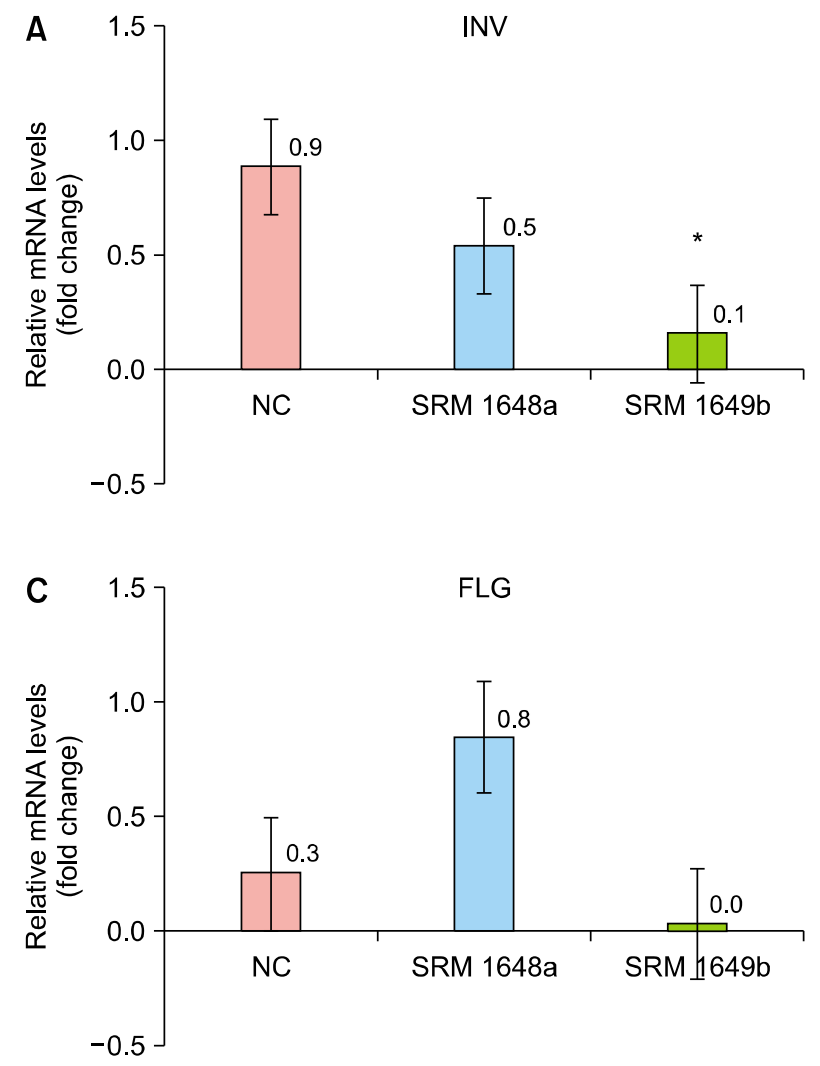

IL-13, CXCL1, CXCL2, CXCL5, MMP13, TNF- $\alpha$, TSLP, TARC, TLR2, TLR4, involucrin, loricrin, filaggrin, claudin-1, ZO-1, and GAPDH. qPCR assays were performed on a real-time thermal cycler (Applied Biosystems; Thermo Fischer Scientific) using PowerUp SYBR Green Master mix (Applied Biosystems; Thermo Fisher Scientific). All results were normalized to the level of GAPDH. Relative quantitation was analyzed using the comparative $\Delta \Delta \mathrm{Ct}$ method according to the manufacturer's instructions.

\section{Western blot analysis}

Protein samples were prepared from the skin tissues, and the $\mathrm{HaCaT}$ cells were cultured with $1 \%$ Triton- $\mathrm{X}$ radioimmunoprecipitation assay buffer containing a protease inhibitor cocktail. The protein concentrations of the lysed cells and homogenized skin tissue samples were determined using the bicinchoninic acid assay. Equal amounts of protein

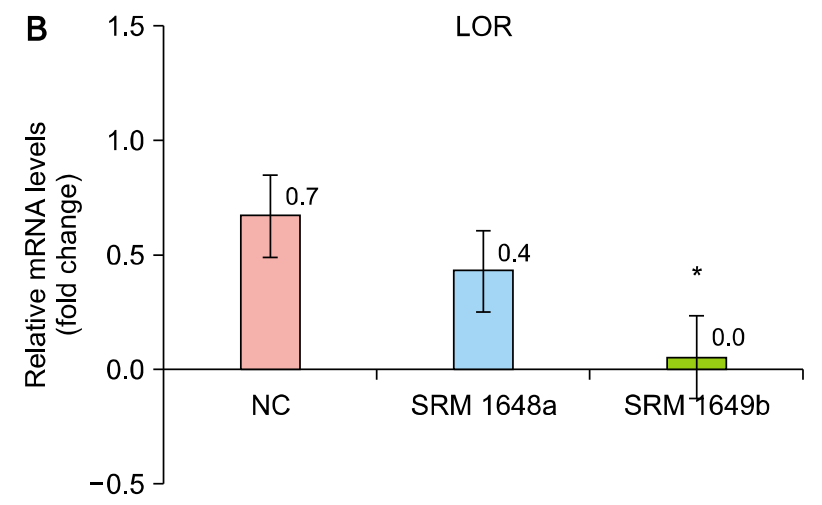

D

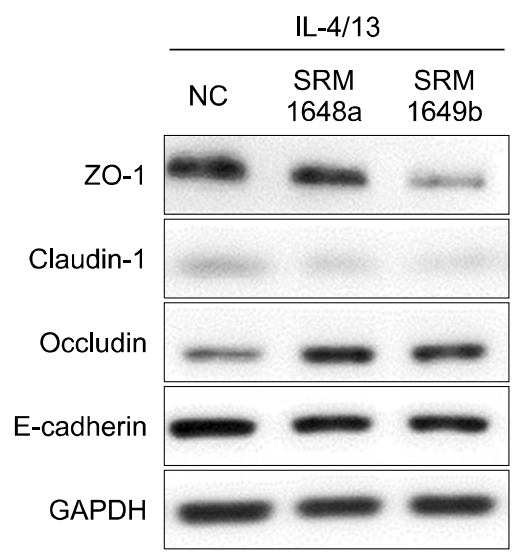

Fig. 2. Standard reference material (SRM) $1649 \mathrm{~b}$ significantly decreased the mRNA expression levels of INV and LOR, and the protein expression of claudin-1 and ZO-1 in HaCaT cells pretreated with interleukin (IL)-4 and IL-13. On the other hand, although SRM 1648a decreased the mRNA expression levels of INV and LOR, the changes were not statistically significant. In general, there was a greater decrease in the mRNA and protein expression levels in HaCaT cells exposed to SRM 1649b than in those exposed to SRM 1648a. The mRNA expression levels of (A) INV, (B) LOR, and (C) FLG in HaCaT cells were determined by real-time quantitative PCR. (D) The protein levels of ZO-1, claudin-1, occludin, and E-cadherin were determined by western blotting. Cells were treated with SRM 1648a $\left(50 \mu \mathrm{g} / \mathrm{cm}^{2}\right)$ and SRM 1649b $\left(25 \mu \mathrm{g} / \mathrm{cm}^{2}\right)$ for 24 hours following IL-4 and IL-13 (50 ng/ml) pre-treatment for 72 hours, respectively. Values are presented as mean \pm standard deviation of two independent experiments $(n=2)$. NC: normal control, INV: involucrin, LOR: loricrin, FLG: filaggrin. ${ }^{*} p<0.05$ vs. NC. 
were loaded onto $8 \% \sim 10 \%$ sodium dodecyl sulfate-polyacrylamide gels. After electrophoresis, the proteins were transferred onto nitrocellulose membranes. The membranes were blocked with 5\% skim milk for 1 hour. Subsequently, the membranes were incubated overnight with primary antibodies at $4^{\circ} \mathrm{C}$; subsequently, they were incubated with horseradish peroxidase-conjugated secondary antibodies for 1 hour at room temperature. Protein expression was detected using the EzWestLumi Plus system (ATTO, Tokyo, Japan), and images were captured by exposing the membranes to a ChemiDoc ${ }^{\mathrm{TM}}$ XRS image analyzer (Bio-Rad, Hercules, CA, USA).

\section{Enzyme-linked immunosorbent assay}

Total IgE levels were analyzed using enzyme-linked immunosorbent assay (ELISA). Blood samples were collected from the intraorbital vein of the mice on sacrifice. The collected whole blood was left undisturbed at room temperature to allow the blood to clot. Serum samples were separated from the whole blood and stored at $-80^{\circ} \mathrm{C}$ until analysis. Total IgE levels were quantified using ELISA kits (Thermo Fisher Scientific) according to the manufacturer's protocol.

\section{Histological examination}

The ear skin of each mouse was fixed in $4 \%$ paraformaldehyde solution. Then, tissues were embedded in paraffin and sliced into 5- $\mu$ m-thick sections; the tissue sections were stained with hematoxylin and eosin (H\&E). Histological changes were examined via light microscopy (Leica D750; Leica Microsystems, Wetzlar, Germany). Means of SC, epidermal, and dermal thickness was quantified by measuring the thickness of 10 randomly selected area of the tissue section with the aid of Image Software $(\mathrm{NIH}$, Bethesda, MD, USA; http://imagej.nih.gov/ij).

\section{Statistical analysis}

Each in vivo assay was performed independently at least twice. Data from each assay are presented as the mean \pm standard deviation (SD). Data were analyzed using oneway or two-way analysis of variance (ANOVA) with the multiple correction test algorithm of Bonferroni. We considered $p$-values $<0.05$ significant. Statistical analysis was performed using SPSS (PASW statistics ver. 18; IBM Corp., Armonk, NY, USA).

\section{RESULTS}

\section{Effects of SRM 1648a and 1649b on HaCaT cells pretreated with IL-4 and IL-13}

First, we investigated the effects of SRM 1648a and 1649b on the SC-related proteins and TJ proteins in HaCaT cells pretreated with IL-4 and IL-13.

As shown in Fig. 2, exposure to SRM 1649b significantly decreased the mRNA expression of involucrin and loricrin and the protein expression of claudin-1 and ZO-1 in HaCaT cells pretreated with IL-4 and IL-13. Exposure to SRM $1649 \mathrm{~b}$ also decreased the mRNA expression of filaggrin; however, the changes were not significant. In contrast, the decreased mRNA expression of involucrin and loricrin with exposure to SRM 1648a was not significant,

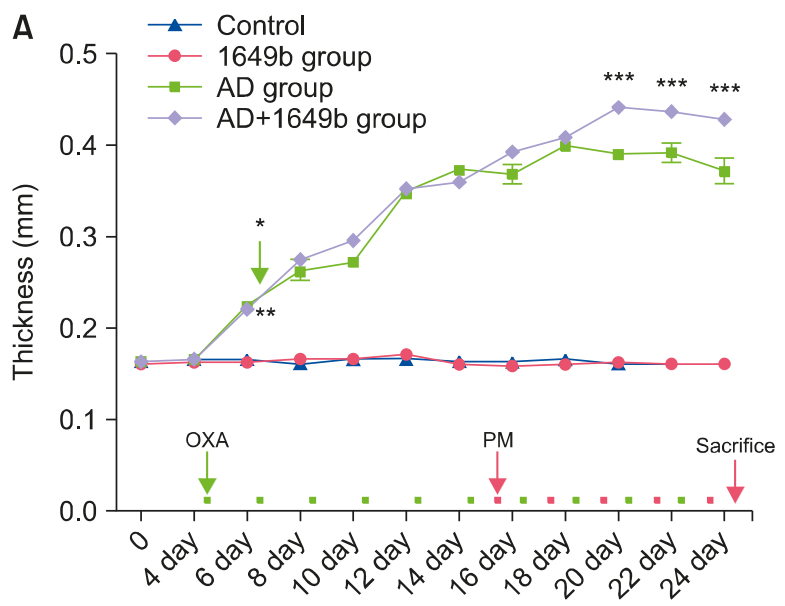

B

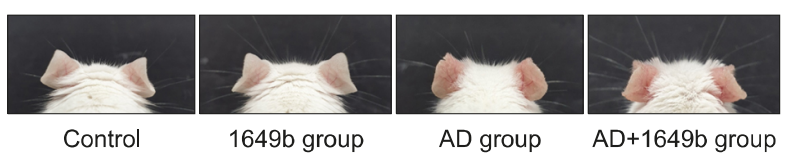

Fig. 3. (A) Repeated exposure to standard reference material (SRM) $1649 \mathrm{~b}$ increased the thickness of the AD-like skin in the oxazolone (OXA)-induced AD-like mouse model. Ear thickness significantly increased in the AD groups. Furthermore, ear thickness was further increased in the $A D+1649 \mathrm{~b}$ group compared to the $A D$ group. Ear thickness was measured using a digital caliper before each OXA challenge and immediately before sacrifice. (B) Exposure to SRM 1649b induced changes in morphology in the OXA-induced AD-like mouse model. Clinical features were observed in the images taken during the repeated application of OXA and SRM 1649b in the mouse model. Values are presented as mean \pm standard deviation of two independent experiments $(n=2)$. AD: atopic dermatitis, PM: particulate matter, 1649 b group: SRM 1649b-exposed control group, $A D+1649 b$ group: SRM 1649b-exposed OXA-induced AD group, AD group: OXAinduced AD group. ${ }^{*} p<0.011649 \mathrm{~b}$ group vs. $A D+1649 \mathrm{~b}$ group. ${ }^{* *} p<0.01$ Control group vs. AD group. ${ }^{* * *} p<0.001$ AD group vs. $A D+1649 b$ group. 
and the mRNA expression of filaggrin was rather increased. However, these changes were not statistically significant. In general, there was a greater decrease in the mRNA and protein expression levels in HaCaT cells exposed to SRM $1649 \mathrm{~b}$ than in those exposed to $1648 \mathrm{a}$. These results indicate that SRM $1649 \mathrm{~b}$ comprising polycyclic aromatic hydrocarbons, polychlorinated biphenyl congeners, and pesticides is more toxic to human keratinocytes than 1648a comprising heavy metals. These results are also consistent with the results of previous studies, which indicated that $1649 \mathrm{~b}$, but not $1648 \mathrm{a}$, significantly disrupted the SC and TJ proteins ${ }^{3}$. Therefore, in the following animal experiments, only SRM 1649b was used.

\section{Effects of SRM 1649b on ear thickness in the mouse model with oxazolone-induced atopic dermatitis-like skin}

To examine the effects of repeated SRM 1649b exposure on skin thickness in the mouse model with OXA-induced AD-like skin lesions, we measured ear thickness using a digital caliper before each OXA challenge (which was performed once every other day) and immediately before sacrifice. There was no significant difference between the normal control and the SRM 1649b-exposed control group (1649b group); however, there was a significant increase in ear thickness in the OXA-induced AD group (AD group) and the SRM 1649b-exposed OXA-induced AD group (AD + 1649b group). In addition, ear thickness was significantly higher in the $A D+1649 b$ group than in the $A D$ group after exposure (three times) to SRM 1649b (Fig. 3).

Histological evaluation further confirmed that the hyperkeratosis and epidermal acanthosis were more pronounced and dermal inflammation was increased in the $A D+1649 b$ group (Fig. 4A). When we measured the thickness of each compartment (SC, epidermis, and dermis), the epidermal and dermal thickness was significantly increased in the $A D+1649 b$ group compared to $A D$ group (Fig. 4B).

\section{Effects of SRM 1649b on inflammatory cytokine, chemokine, and toll-like receptor production in the mouse model with oxazolone-induced atopic dermatitis-like skin}

To investigate the effects of SRM $1649 \mathrm{~b}$ on inflammatory

A

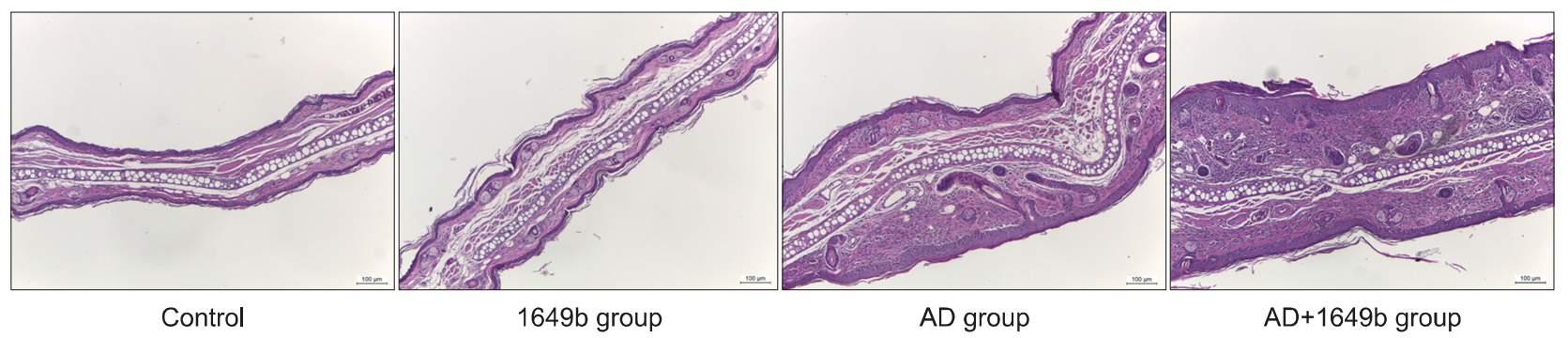

B
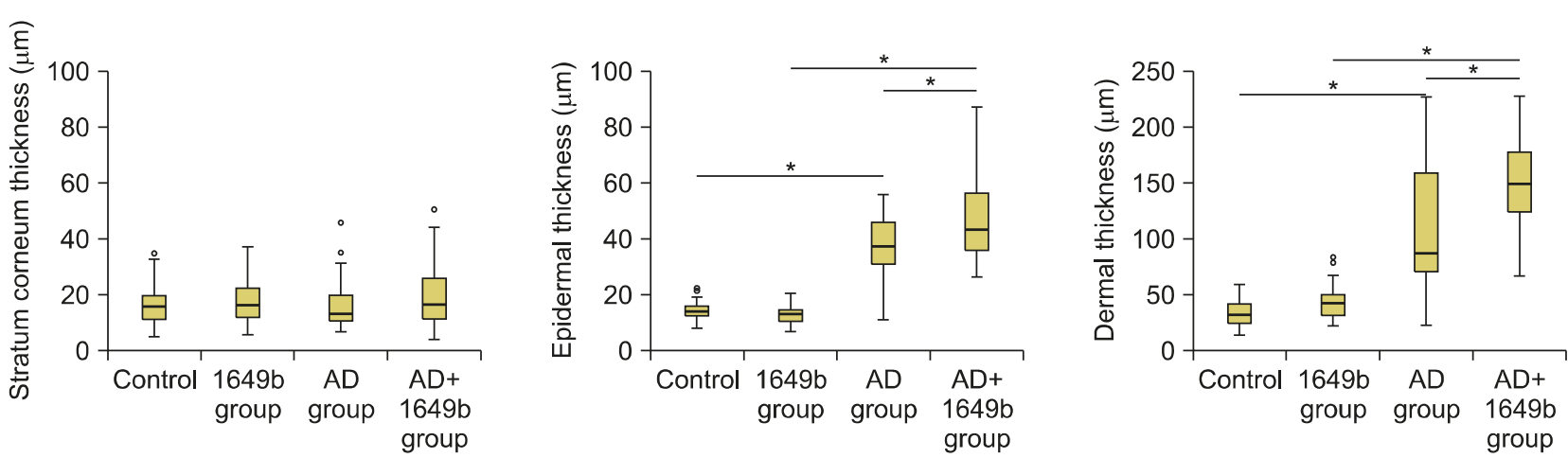

Fig. 4. (A) Histological examination confirmed that epidermal and dermal thickness were increased with dermal inflammatory cell infiltration in the $A D+1649 b$ group compared to the $A D$ group $(H \& E$, Scale bar $=100 \mu \mathrm{m})$. Hematoxylin and eosin stain. (B) The epidermal and dermal thickness was significantly increased in the AD+1649b group compared to AD group. The thicknesses of each compartment (stratum corneum, epidermis, and dermis) were quantified by measuring the thickness of 10 randomly selected area of the tissue section with the aid of ImageJ Software (NIH, Bethesda, MD, USA; http://imagej.nih.gov/ij). AD: atopic dermatitis, 1649b group: standard reference material (SRM) 1649b-exposed control group, AD+1649b group: SRM 1649b-exposed oxazolone (OXA)-induced $\mathrm{AD}$ group, $\mathrm{AD}$ group: OXA-induced $\mathrm{AD}$ group. ${ }^{*} p<0.05$ using ANOVA with the multiple correction test algorithm of Bonferroni. 
responses in OXA-induced $\mathrm{AD}$-like skin lesions, we determined the levels of inflammatory cytokines, chemokines, and toll-like receptor using qPCR. Although mice do not have a human IL-8 homolog, CXCL1 (KC), CXCL2 (MIP-2), and CXCL5 (LIX) are recognized as functional homologs ${ }^{17,34}$. Therefore, the mRNA levels of IL- $1 \alpha, \mathrm{IL}-1 \beta$, IL-4, IL-6, IL-13, CXCL1 (KC), CXCL2 (MIP-2), CXCL5 (LIX), TNF- $\alpha$, TSLP, TARC, TLR2, TLR4, and MMP13 were analyzed.

The mRNA levels of MMP13, CXCL1, CXCL2, and CXCL5 significantly increased in the $A D$ group compared to the control and $1649 \mathrm{~b}$ group. Furthermore, subsequent PM exposure increased all four mRNA levels, and in particular the mRNA levels of CXCL1 and CXCL5 significantly increased in $A D+1649 b$ compared to AD group (Fig. 5). There was no statistically significant difference in the
mRNA expression levels of other inflammatory cytokines, chemokines, and toll-like receptor between the groups.

\section{Effects of SRM 1649b on stratum corneum-related protein and tight junction protein expression in the mouse model with oxazolone-induced atopic dermatitis-like skin}

To investigate the effects of SRM $1649 \mathrm{~b}$ on the expression of SC-related proteins and TJ proteins in the mouse model with OXA-induced AD-like skin lesions, we analyzed the expression levels of involucrin, loricrin, filaggrin, claudin-1, and ZO-1 using qPCR and western blot analysis. As presented in Fig. 6, SRM 1649b exposure reduced the mRNA and protein expression levels of filaggrin, involucrin, loricrin, claudin-1, and ZO-1 in both the control and OXA-induced AD groups. However, the decrease in
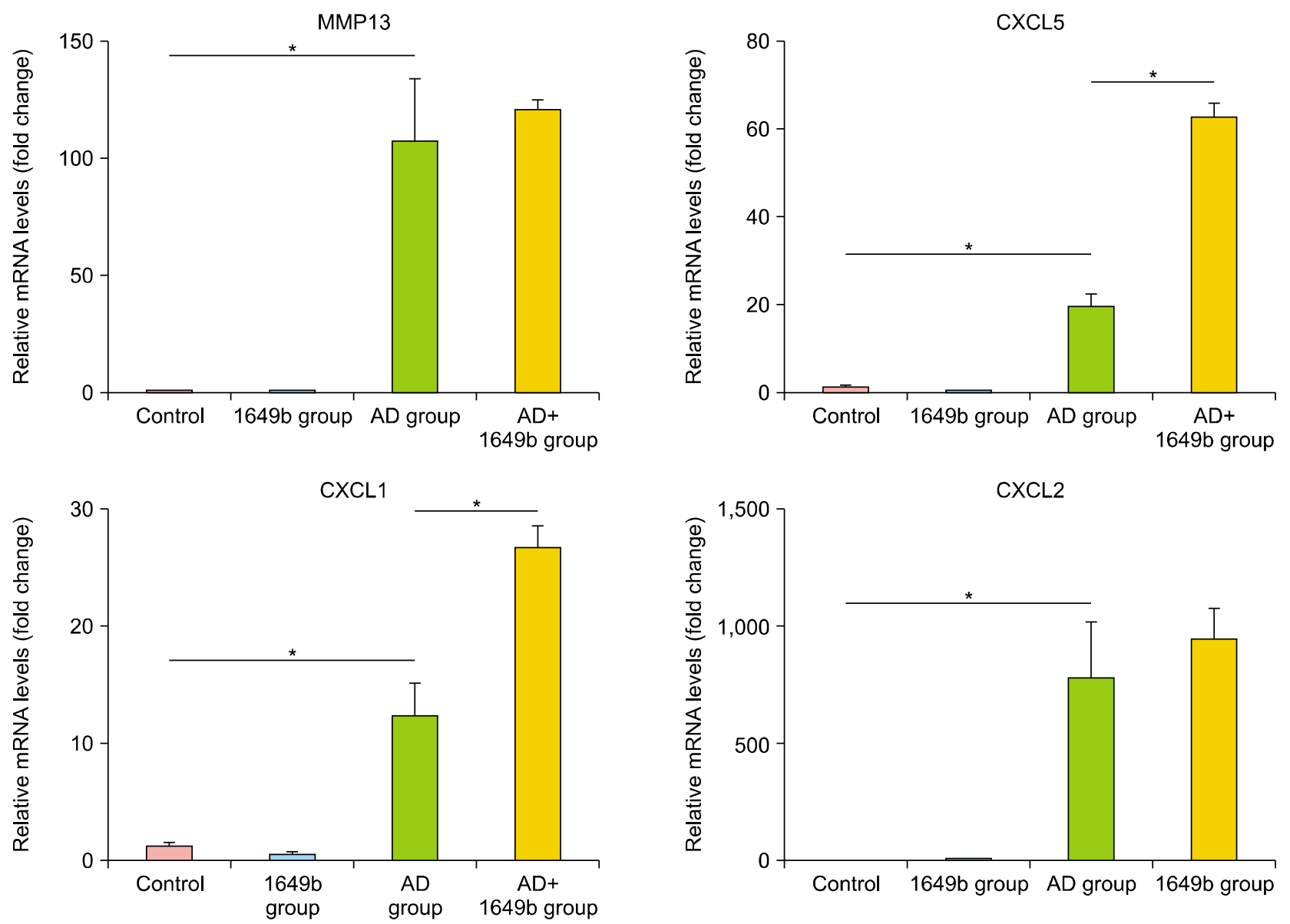

Fig. 5. Effects of standard reference material (SRM) $1649 \mathrm{~b}$ on the inflammatory response in oxazolone (OXA) induced dermatitis-like mice model. The mRNA levels of MMP13, CXCL1, CXCL2, and CXCL5 significantly increased in the atopic dermatitis (AD) group compared to the control and $1649 \mathrm{~b}$ group. The mRNA levels of CXCL1 and CXCL5 significantly increased in AD $+1649 \mathrm{~b}$ compared to $\mathrm{AD}$ group. The mRNA expression of were measured by real-time quantitative PCR. The relative mRNA and protein expression levels in AD-like mice model are shown. These levels were normalized to those of $\beta$-actin. Values are presented as mean \pm standard deviation of two independent experiments $(n=2)$. 1649b group: SRM 1649b-exposed control group, AD $+1649 b$ group: SRM 1649b-exposed OXA-induced AD group, AD group: OXA-induced AD group. ${ }^{*} p<0.05$. 

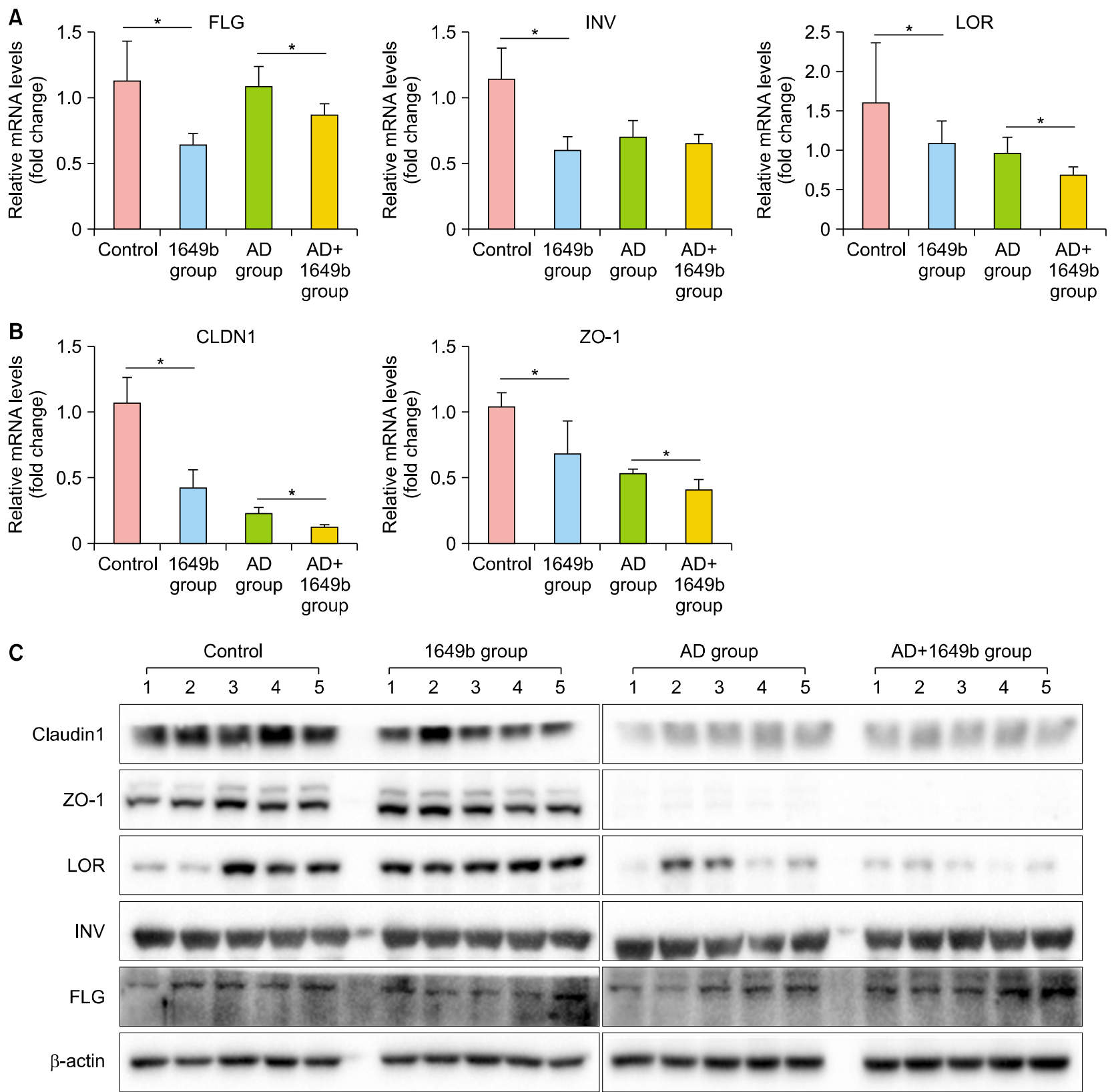

Fig. 6. Standard reference material (SRM) 1649b exposure reduced the mRNA and protein expression levels of FLG, INV, LOR, CLDN-1, and ZO-1 in both control and oxazolone (OXA)-induced atopic dermatitis (AD) groups. However, the decrease in the expression of the stratum corneum-related proteins and tight junction proteins after SRM exposure was comparable between the two groups. The mRNA and protein levels of skin barrier-related proteins were analyzed using (A, B) real-time quantitative PCR and (C) western blotting, respectively. The relative mRNA and protein expression levels in the AD-like mouse model are shown. These levels were normalized to the mRNA expression levels of $\beta$-actin. Values are presented as mean \pm standard deviation of two independent experiments $(n=2)$. FLG: filaggrin, INV: involucrin, LOR: loricrin, CLDN-1: claudin-1, 1649b group: SRM 1649b-exposed control group, AD +1649 b group: SRM 1649b-exposed OXA-induced AD group, AD group: OXA-induced AD group. ${ }^{*} p<0.05$.

the expression of the SC-related proteins and TJ, proteins after SRM 1649b exposure was comparable between the two groups. These results suggest that PM downregulates the expression of SC-related proteins and TJ proteins in both healthy and AD skin.
Effects of SRM 1649b on immunoglobulin E production in the mouse model with oxazolone-induced atopic dermatitis-like skin

To investigate whether SRM $1649 \mathrm{~b}$ induces IgE production in mice, we determined the serum IgE levels using 
ELISA analysis. For ELISA analysis, serum was isolated from the collected whole blood of the mice. The serum IgE levels were higher in the OXA-induced AD groups (AD group and $A D+1649 b$ group) than in the control group. However, there was no significant difference in the serum IgE levels between the AD group and the $A D+1649 b$ group (data not shown).

\section{DISCUSSION}

In this study, we investigated whether PM aggravates ADlike symptoms by evaluating the epidermal thickness and histological changes in a mouse model with OXA-induced AD-like skin. We also investigated whether PM affects the expression levels of SC-related proteins and TJ proteins because the decreased expression of these proteins is related to various skin disorders and can cause skin barrier dysfunction.

As shown in Fig. 3, we found that the ear thickness of the mouse model with AD-like skin was significantly increased after exposure to PM. Histological evaluation further confirmed significant increase in the epidermal and dermal thickness with heavy dermal cell infiltration after PM exposure in AD-like skin. These results show that PM exposure clinically exacerbates AD symptoms characterized by epidermal acanthosis and dermal inflammation.

Recently, the focus on the pathogenesis of AD has evolved from concentration on immune system dysfunction as the primary abnormality in disease development to the incorporation of the concept of epidermal barrier dysfunc$\operatorname{tion}^{35}$. Numerous alterations and deficiencies in the SC-related proteins and TJ proteins in the skin of atopic individuals have been implicated in the disturbed skin barrier, highlighting the importance of the skin barrier in disease development. For instance, in both the lesional and nonlesional skin of patients with $A D$, filaggrin mutation has been shown to contribute to AD pathogenesis. Loricrin and involucrin, two other important proteins that facilitate the terminal differentiation of the epidermis and the formation of the skin barrier, are known to be downregulated in $\mathrm{AD} \operatorname{skin}^{36}$. Furthermore, humans with filaggrin mutations show decreased expression of TJ proteins such as occludin and $\mathrm{ZO}-1^{37}$. In addition, reduced expression of the TJ protein claudin-1 has also been demonstrated in the nonlesional skin of individuals with $\mathrm{AD}^{38}$.

The results of our study showed that PM downregulates the expression of SC-related proteins and TJ proteins in both control and AD skin. These results are also consistent with those of previous studies. Pan et al. ${ }^{3}$ have shown that SRM 1649b disrupted the SC and TJs in pig skin. Some proteins related to the barrier function, such as cytoker- atin, filaggrin, and E-cadherin, were also diminished after $1649 \mathrm{~b}$ treatment. Lehmann et al. ${ }^{39}$ have also shown that PMs damage the TJs of the lung epithelial barrier. Lee et al. $^{16}$ found that PM induced the downregulation of filaggrin protein and mRNA expression in a time- and dose-dependent manner in human keratinocytes via cyclooxygenase 2 expression and prostaglandin E2 production. However, all these previous studies have only evaluated the effects of PM on the skin barrier function in non-AD, normal, intact skin. None of the previous studies have evaluated the effects of PM on the skin barrier function in AD skin.

Our results suggest that PM exposure disrupts normal keratinocyte differentiation in both normal and AD skin. These effects were neither more specific nor greater in AD skin than in normal intact skin. However, in AD skin, where the skin barrier is already impaired, the disruption in normal keratinocyte differentiation would halt barrier repair and further aggravate skin barrier dysfunction. For instance, deregulation of keratinocyte differentiation and activation markers have been implicated in the non-healing edges of patients with venous ulcer $^{40}$. Similarly, PM exposure, which leads to the deregulation of keratinocyte differentiation, would disturb the normal healing process of the impaired skin barrier in patients with AD.

The impaired skin barrier would allow further allergen exposure, which subsequently drives inflammation and the release of cytokines, which, in turn, reduce the skin barrier function, leading to a vicious cycle ${ }^{41,42}$. In our previous study, we showed that PM exposure can induce the expression of pro-inflammatory cytokines in $\mathrm{HaCaT}$ cells when the cells are directly treated with $\mathrm{PM}^{29}$. In this study, we found that the mRNA levels of functional homologues of human IL-8 cytokine (CXCL1, CXCL2, and CXCL5) and MMP13 increased following PM exposure in AD skin, although statistically significant changes were observed only in CXCL1 and CXCL5. These results are in concordance with previous reports by Jin et al. ${ }^{17}$ where they found that the mRNA levels of CXCL1, CXCL2, CXCL5, and MMP13 were significantly increased when PM was directly applied with transparent film dressing on the tape-stripped dorsal skin of mice for 6 consecutive days. Although similar results were observed in our study, statistical significances were only achieved for CXCL1 and CXCL5. This may be due to the limited exposure to $\mathrm{PM}$ in our study (mice were exposed to $100 \mu \mathrm{g} / \mathrm{cm}^{3} \mathrm{PM}$ for $1 \mathrm{~h} /$ day, once every other day for 10 days), which may not have been enough to experimentally prove the changes in the inflammatory cytokines and chemokines in the in vivo mouse model.

There are several limitations of this study. First, regarding 
the skin barrier function, we did not evaluate the effect of PM on intercellular lipids of SC including free fatty acids, ceramides, and cholesterol. It is well known that the composition of lipids in SC is important on the integrity of the SC and previous studies have shown that in patients with $A D$, ceramide levels were lowered and cholesterol was increased in $\mathrm{SC}^{43}$. Furthermore, a few epidemiological studies have shown that people living in heavily polluted areas had higher sebum levels and a lower ratio of squalene/cholesterol ${ }^{44,45}$. More importantly, a recent study using transcriptomic analysis has shown that PM2.5 could disturb cholesterol homeostasis, and 3-dimensional in vitro skin model treated with PM2.5 showed that the cholesterol levels were much higher than that of the control group $^{46}$. Thus, PM can impair skin barrier function by interfering with intercellular lipids. However, it should also be noted that barrier disruption itself induces lipid synthesis to promote repair of the skin barrier. Since cholesterol is an important component of cell membranes, damaged skin accelerates the rate of cell proliferation and a large amount of cholesterol is synthesized to supplement keratinocyte, intercellular lipids and repair the damage $\mathrm{SC}^{47}$. Therefore, it is still unclear whether PM directly impairs homeostasis of intercellular lipids of SC. The impaired cholesterol homeostasis may be the compensatory response of the damaged skin barrier to repair itself. Thus, future studies should be performed to elucidate this. Second, although we observed increase in dermal inflammation in the tissue section of $A D+1649 b$ skin, the changes in inflammatory cytokines and chemokines were inconsistent following PM exposure. This may be due to the effect of PM directly penetrating into the dermis of barrier-damaged skin as shown by Jin et $\mathrm{al}^{17}$. To elucidate this, further evaluation for gene expressions of NF- $\kappa$ B and quantifying dermal inflammation by immunohistochemical staining for myeloperoxidase are required in the future studies.

In conclusion, we investigated the effects of $P M$ on $A D$ skin using $\mathrm{HaCaT}$ cells and a mouse model with AD-like skin. Our results showed that PM exposure decreases the expression levels of SC-related proteins and TJ proteins. Clinically, we found that PM exposure significantly increased the epidermal and dermal thickness of the ears of the model mice with AD-like skin (Fig. 3). Based on these findings, we suggest that $P M$ can aggravate $A D$ symptoms, and that this may be related to the negative effects of PM on the skin barrier function. Therefore, further research should be performed to elucidate the detailed mechanism and biological crosstalk between the epidermis and dermis, when AD skin is exposed to PM.

\section{CONFLICTS OF INTEREST}

The authors have nothing to disclose.

\section{FUNDING SOURCE}

This work was supported by the National Research Foundation of Korea (NRF) grant funded by the Korea government (MSIP; Ministry of Science, ICT \&Future Planning) (No. NRF-2017R1C1B5017825).

\section{DATA SHARING STATEMENT}

The data that support the findings of this study are available from the corresponding author upon reasonable request.

\section{ORCID}

Yoo Jung Bae, https://orcid.org/0000-0001-8861-600X

Kui Young Park, https://orcid.org/0000-0001-5965-1754

Hye Sung Han, https://orcid.org/0000-0002-3556-0740

Young Shin Kim, https://orcid.org/0000-0002-5928-1201

Ji Yeon Hong, https://orcid.org/0000-0002-5632-8449

Tae Young Han, https://orcid.org/0000-0003-0317-9635

Seong Jun Seo, https://orcid.org/0000-0003-2915-839X

\section{REFERENCES}

1. Kim KE, Cho D, Park HJ. Air pollution and skin diseases: adverse effects of airborne particulate matter on various skin diseases. Life Sci 2016;152:126-134.

2. Lee CW, Lin ZC, Hsu LF, Fang JY, Chiang YC, Tsai MH, et al. Eupafolin ameliorates COX-2 expression and PGE2 production in particulate pollutants-exposed human keratinocytes through ROS/MAPKs pathways. J Ethnopharmacol 2016; 189:300-309.

3. Pan TL, Wang PW, Aljuffali IA, Huang CT, Lee CW, Fang JY. The impact of urban particulate pollution on skin barrier function and the subsequent drug absorption. J Dermatol Sci 2015;78:51-60.

4. Kappos AD, Bruckmann P, Eikmann T, Englert N, Heinrich $U$, Höppe $P$, et al. Health effects of particles in ambient air. Int J Hyg Environ Health 2004;207:399-407.

5. Anderson JO, Thundiyil JG, Stolbach A. Clearing the air: a review of the effects of particulate matter air pollution on human health. J Med Toxicol 2012;8:166-175.

6. Mancebo SE, Wang SQ. Recognizing the impact of ambient air pollution on skin health. J Eur Acad Dermatol Venereol 2015;29:2326-2332.

7. Martinelli N, Olivieri O, Girelli D. Air particulate matter and cardiovascular disease: a narrative review. Eur J Intern Med 2013;24:295-302.

8. Brook RD, Rajagopalan S, Pope CA 3rd, Brook JR, Bhatnagar 
A, Diez-Roux AV, et al. Particulate matter air pollution and cardiovascular disease: an update to the scientific statement from the American Heart Association. Circulation 2010;121: 2331-2378.

9. Krutmann J, Liu W, Li L, Pan X, Crawford M, Sore G, et al. Pollution and skin: from epidemiological and mechanistic studies to clinical implications. J Dermatol Sci 2014;76:163168.

10. Hänel KH, Cornelissen C, Lüscher B, Baron JM. Cytokines and the skin barrier. Int J Mol Sci 2013;14:6720-6745.

11. Rawlings AV, Harding CR. Moisturization and skin barrier function. Dermatol Ther 2004;17(Suppl 1):43-48.

12. Wickett RR, Visscher MO. Structure and function of the epidermal barrier. Am J Infect Control 2006;34(Suppl 2): S98-S110.

13. Egawa G, Kabashima K. Barrier dysfunction in the skin allergy. Allergol Int 2018;67:3-11.

14. Li Q, Kang Z, Jiang S, Zhao J, Yan S, Xu F, et al. Effects of ambient fine particles PM2.5 on human HaCaT cells. Int J Environ Res Public Health 2017;14:72.

15. Zhao R, Guo Z, Zhang R, Deng C, Xu J, Dong W, et al. Nasal epithelial barrier disruption by particulate matter $\leq$ $2.5 \mathrm{~cm}$ via tight junction protein degradation. J Appl Toxicol 2018;38:678-687.

16. Lee CW, Lin ZC, Hu SC, Chiang YC, Hsu LF, Lin YC, et al. Urban particulate matter down-regulates filaggrin via COX2 expression/PGE2 production leading to skin barrier dysfunction. Sci Rep 2016;6:27995.

17. Jin SP, Li Z, Choi EK, Lee S, Kim YK, Seo EY, et al. Urban particulate matter in air pollution penetrates into the barrierdisrupted skin and produces ROS-dependent cutaneous inflammatory response in vivo. J Dermatol Sci 2018;91:175183.

18. Lademann J, Schaefer H, Otberg N, Teichmann A, BlumePeytavi U, Sterry W. [Penetration of microparticles into human skin]. Hautarzt 2004;55:1117-1119. German.

19. Ahn K. The role of air pollutants in atopic dermatitis. J Allergy Clin Immunol 2014;134:993-999; discussion 1000.

20. Cork MJ, Danby SG, Vasilopoulos Y, Hadgraft J, Lane ME, Moustafa $M$, et al. Epidermal barrier dysfunction in atopic dermatitis. J Invest Dermatol 2009;129:1892-1908.

21. Kim JY, Jeong MS, Park MK, Lee MK, Seo SJ. Time-dependent progression from the acute to chronic phases in atopic dermatitis induced by epicutaneous allergen stimulation in NC/Nga mice. Exp Dermatol 2014;23:53-57.

22. Jung $Y$, Kim JC, Park NJ, Bong SK, Lee $S$, Jegal $H$, et al. Eupatilin, an activator of PPARa, inhibits the development of oxazolone-induced atopic dermatitis symptoms in Balb/c mice. Biochem Biophys Res Commun 2018;496:508-514.

23. Morgenstern V, Zutavern A, Cyrys J, Brockow I, Koletzko S, Krämer $U$, et al. Atopic diseases, allergic sensitization, and exposure to traffic-related air pollution in children. Am J Respir Crit Care Med 2008;177:1331-1337.

24. Kim J, Kim EH, Oh I, Jung K, Han Y, Cheong HK, et al. Symptoms of atopic dermatitis are influenced by outdoor air pollution. J Allergy Clin Immunol 2013;132:495-498.e1.

25. Huss-Marp J, Eberlein-König B, Breuer K, Mair S, Ansel A,
Darsow $U$, et al. Influence of short-term exposure to airborne Der $\mathrm{p} 1$ and volatile organic compounds on skin barrier function and dermal blood flow in patients with atopic eczema and healthy individuals. Clin Exp Allergy 2006; 36:338-345.

26. Pénard-Morand C, Raherison C, Charpin D, Kopferschmitt C, Lavaud F, Caillaud D, et al. Long-term exposure to closeproximity air pollution and asthma and allergies in urban children. Eur Respir J 2010;36:33-40.

27. Suárez-Varela MM, Gallardo-Juan A, García-Marcos L, GimenoClemente N, Silvarrey-Varela AL, Miner-Canflanca I, et al. The impact of atmospheric pollutants on the prevalence of atopic eczema in 6-7-year-old schoolchildren in Spain; ISAAC Phase III. Iran J Allergy Asthma Immunol 2013;12: 220-227.

28. Kim HO, Kim JH, Cho SI, Chung BY, Ahn IS, Lee $\mathrm{CH}$, et al. Improvement of atopic dermatitis severity after reducing indoor air pollutants. Ann Dermatol 2013;25:292-297.

29. Kim M, Kim JH, Jeong GJ, Park KY, Lee MK, Seo SJ. Particulate matter induces pro-inflammatory cytokines via phosphorylation of p38 MAPK possibly leading to dermal inflammaging. Exp Dermatol 2019;28:809-815.

30. Kim JH, Kim M, Kim JM, Lee MK, Seo SJ, Park KY. Afzelin suppresses proinflammatory responses in particulate matterexposed human keratinocytes. Int J Mol Med 2019;43:25162522.

31. Jin H, He R, Oyoshi M, Geha RS. Animal models of atopic dermatitis. J Invest Dermatol 2009;129:31-40.

32. Man MQ, Hatano $Y$, Lee $S H$, Man M, Chang S, Feingold $K R$, et al. Characterization of a hapten-induced, murine model with multiple features of atopic dermatitis: structural, immunologic, and biochemical changes following single versus multiple oxazolone challenges. J Invest Dermatol 2008; 128:79-86.

33. Kim SY, Kim JK, Park SH, Kim BG, Jang AS, Oh $\mathrm{SH}$, et al. Effects of inhaled particulate matter on the central nervous system in mice. Neurotoxicology 2018;67:169-177.

34. Kim BG, Lee PH, Lee SH, Kim YE, Shin MY, Kang Y, et al. Long-term effects of diesel exhaust particles on airway inflammation and remodeling in a mouse model. Allergy Asthma Immunol Res 2016;8:246-256.

35. Hanifin JM. Evolving concepts of pathogenesis in atopic dermatitis and other eczemas. J Invest Dermatol 2009;129: 320-322.

36. Kim BE, Leung DY, Boguniewicz M, Howell MD. Loricrin and involucrin expression is down-regulated by Th2 cytokines through STAT-6. Clin Immunol 2008;126:332-337.

37. Gruber R, Elias PM, Crumrine D, Lin TK, Brandner JM, Hachem JP, et al. Filaggrin genotype in ichthyosis vulgaris predicts abnormalities in epidermal structure and function. Am J Pathol 2011;178:2252-2263.

38. De Benedetto A, Rafaels NM, McGirt LY, Ivanov Al, Georas $\mathrm{SN}$, Cheadle C, et al. Tight junction defects in patients with atopic dermatitis. J Allergy Clin Immunol 2011;127:773-786. e1-7.

39. Lehmann AD, Blank F, Baum O, Gehr P, Rothen-Rutishauser BM. Diesel exhaust particles modulate the tight junction 
protein occludin in lung cells in vitro. Part Fibre Toxicol 2009;6:26.

40. Stojadinovic O, Pastar I, Vukelic S, Mahoney MG, Brennan D, Krzyzanowska A, et al. Deregulation of keratinocyte differentiation and activation: a hallmark of venous ulcers. J Cell Mol Med 2008;12:2675-2690.

41. Shreffler WG, Castro RR, Kucuk ZY, Charlop-Powers Z, Grishina G, Yoo $S$, et al. The major glycoprotein allergen from Arachis hypogaea, Ara $\mathrm{h} \mathrm{1}$, is a ligand of dendritic cell-specific ICAM-grabbing nonintegrin and acts as a Th2 adjuvant in vitro. J Immunol 2006;177:3677-3685.

42. Traidl-Hoffmann C, Mariani V, Hochrein H, Karg K, Wagner $\mathrm{H}$, Ring J, et al. Pollen-associated phytoprostanes inhibit dendritic cell interleukin-12 production and augment $\mathrm{T}$ helper type 2 cell polarization. J Exp Med 2005;201:627-636.

43. Di Nardo A, Wertz P, Giannetti A, Seidenari S. Ceramide and cholesterol composition of the skin of patients with atopic dermatitis. Acta Derm Venereol 1998;78:27-30.

44. Lefebvre MA, Pham DM, Boussouira B, Bernard D, Camus C, Nguyen QL. Evaluation of the impact of urban pollution on the quality of skin: a multicentre study in Mexico. Int J Cosmet Sci 2015;37:329-338.

45. Lefebvre MA, Pham DM, Boussouira B, Qiu H, Ye C, Long $\mathrm{X}$, et al. Consequences of urban pollution upon skin status. A controlled study in Shanghai area. Int J Cosmet Sci 2016;38:217-223.

46. Liao Z, Nie J, Sun P. The impact of particulate matter (PM2.5) on skin barrier revealed by transcriptome analysis: focusing on cholesterol metabolism. Toxicol Rep 2019;7: 1-9.

47. Proksch E, Fölster-Holst R, Jensen JM. Skin barrier function, epidermal proliferation and differentiation in eczema. J Dermatol Sci 2006;43:159-169. 